\title{
Association Rule Mining for Product Selling Tactics with High Confidence
}

\author{
Vaibhav E. Pawar \\ Assistant Professor, \\ Information Technology Department \\ Bharati Vidyapeeth College of Engineering, \\ Navi Mumbai.
}

\begin{abstract}
This Mining rules help information proprietors to disclose concealed examples from their information to break down and foresee the procedure on application space. Be that as it may, mining rules in an appropriated domain differentially private is certainly not a minor undertaking because of protection concerns. Information proprietors are keen on teaming up to mine principles on various levels; notwithstanding, they are worried that delicate data identified with the someone engaged with their database may get traded off during the mining procedure. Here plan and figure the issue of illuminating association rules inquiries in a situation with the end goal that the mining procedure is classified and the results are differentially private. Work proposes a protection safeguarding association rules mining where solid association rules are resolved secretly, and the outcomes returned fulfill differential security. At long last done trials on genuine information it shows that planned methodology can productively answer association rules inquiries and is adaptable with expanding information records.
\end{abstract}

Keywords-Association rules mining, Data Privacy, Data Mining, High confidence.

\section{INTRODUCTION}

Due to the quick progression of information assortment and capacity advances, separating information and concealed examples from put away information has become a significant need for people, organizations, and government offices. Regardless, separate data is viewed as a test when the information is disseminated over different proprietors, and every datum proprietor is worried about the security of people in his information. For example, organizations may be keen on acquiring data concerning the budgetary status of people from various items and deal cost. Security Preserving Data Mining (PPDM) procedures has been used with regards to appropriated processing to ensure the classification of the information of every supplier, while as yet empowering the suppliers to perform information mining undertakings, for example, visit itemsets mining and association rules mining, on the disseminated information.

This work portrays a security shielding approach for rules mining. Three sorts of individuals are normal in the proposed model: information suppliers, ace excavator, and information shoppers. The data being shared is as a table that is on a level plane separated into sub-tables, all of which is encouraged by one data provider. Proposed structure safeguard the security of every supplier's selling information while additionally ensuring the inquiry classification against the information suppliers.

\author{
Dipali T. Yadav \\ Assistant Professor, \\ Information Technology Department \\ MGM Engineering Kalamboli.
}

The point of this work can be abridged as follows:

1. Design a security saving methodology for noting association rules questions with the objective of safeguarding the two information protection and high certainty.

2. Our proposed way to deal with gives the differentially private association rules.

3. The proposed technique safeguards the protection of the mined information by keeping every datum supplier from learning delicate data about other information suppliers during the mining procedure.

4. Conduct execution assessment on genuine information to contemplate the versatility and productivity of our model.

\section{RELATED WORK}

Association rule mining is imperative to getting appropriate relationships result inside huge datasets. Association rule mining is identified with the successive thing set mining issue which decides sets of things that show up as often as possible in a dataset. An association rule $r$ is a ramifications of the structure $\mathrm{X} \rightarrow \mathrm{Y}$, where $\mathrm{X}, \mathrm{Y} \subseteq \mathrm{I}$ are thing sets, which catches the idea that an exchange that contains $X$ likewise contains $\mathrm{Y}$. The quality of an association rule is estimated by its certainty, characterized as

$$
\mathrm{c}(\mathrm{X} \rightarrow \mathrm{Y})=\sigma(\mathrm{X} \cup \mathrm{Y}) / \sigma(\mathrm{X}) .
$$

The help of the standard, characterized as $\sigma(\mathrm{XUY})$, is a pointer of the factual centrality of the standard. Commonly, for a standard to be delegate, its recurrence must surpass a base help limit.

Likewise, Association rule mining has numerous preferences; mining results make mystery of touchy data about people remembered for the dataset. For example, with some foundation information on the things bought by any individual from a market client in a given day, an enemy might have the option to limit that specific individual exchange to a little set, and find out about different things, possibly touchy, that she may have purchased. These issues have been first distinguished in, and various arrangements have been proposed since, finishing with the cutting edge and 
provably makes sure about procedures for differentially private information mining.

Differential security is an assurance model that limits the likelihood of a foe to realize whether a specific individual is available in the dataset or not. To accomplish this objective, Differential security permits just factual inquiries to the information, and the consequence of each inquiry is hazardous with arbitrary commotion. Existing best in class differential protection consistent mining procedures follow a Frequent Item set Mining-driven methodology: first, they process loud backings for countless thing sets, and afterward they distinguish high-certainty association rules dependent on thing set backings. In any case, this system just functions admirably for rules with exceptionally enormous backings. For lower-bolster thing sets, the measure of clamor added prompts huge mistakes in the calculation of certainty. Truth be told, to evade huge mistakes, the best in class PrivBasis procedure doesn't register thing set backings for moderate and low recurrence thing sets. some datasets PrivBasis disposes of itemsets and relating association decides that happen in less than half everything being equal.

As indicated by creators Mihai Maruseac and Gabriel Ghinita [1], Association rule mining (ARM) was basic in finding relationships inside huge datasets. ARM was identified with the Frequent Item set Mining (FIM) issue which decides sets of things (i.e., thing sets) that show up as often as possible in a dataset. An association rule $r$ was a ramifications of the structure $\mathrm{X} \rightarrow \mathrm{Y}$, where $\mathrm{X}, \mathrm{Y} \subseteq \mathrm{I}$ was thing sets, which catches the idea that an exchange that contains $\mathrm{X}$ additionally contains $\mathrm{Y}$. The quality of an association rule was estimated by its certainty, characterized as $\mathrm{c}(\mathrm{X} \rightarrow \mathrm{Y})=\sigma(\mathrm{X} \cup \mathrm{Y}) / \sigma(\mathrm{X})$ . The help of the standard, characterized as $\sigma(X \cup Y)$, was a pointer of the factual centrality of the standard. Regularly, for a standard to be delegate, its recurrence must surpass a base help edge. Despite the fact that ARM has various advantages, mining results may reveal touchy insights regarding people remembered for the dataset. For example, with some foundation information on the things bought by Alice (a general store client) in a given day, an enemy might have the option to limit Alice exchange to a little set, and find out about different things, possibly touchy, that she may have purchased. This risk has been first recognized in, and various arrangements have been proposed since, finishing with the cutting edge and provably makes sure about systems for differentially private information mining.

As per Arik Friedman and Assaf Schuster [2], Differential security (DP) was an insurance model that limits the likelihood of a foe to realize whether a specific individual is available in the dataset or not. To accomplish this objective, DP permits just factual inquiries to the information, and the consequence of each inquiry is irritated with arbitrary commotion. Existing cutting edge DP-consistent mining strategies follow a FIM-driven methodology: first, they register uproarious backings for countless thing sets, and afterward they distinguish high-certainty association rules dependent on thing set backings. Be that as it may, this technique just functions admirably for rules with exceptionally enormous backings. For lower-bolster thing sets, the measure of clamor added prompts huge blunders in the calculation of certainty. Truth be told, to keep away from enormous mistakes, the best in class PrivBasis system doesn't process thing set backings for moderate-and low-recurrence thing sets. Some datasets PrivBasis disposes of thing sets and comparing association decides that happen in less than half everything being equal.

As per the Rakesh Agrawal and Ramkrishnat Shrikant [3], the issue of security sparing data assessment has a long history spreading over different controls. As electronic data about individuals ends up being continuously low down, and as advancement engages constantly unfathomable collection and curation of these data, the need increases for a solid, critical, and numerically intensive importance of security, together with a computationally rich class of figurings that satisfy this definition. Differential Privacy is such a definition. In the wake of convincing and discussing the noteworthiness of differential insurance, the predominance of this monograph is given to head techniques for achieving differential security, and utilization of these methods in imaginative blends, using the request release issue as an Ongoing model.

A key point was that, by reconsidering the computational objective, one can regularly get obviously better outcomes than would be accomplished b effectively superseding every movement of a non-private estimation with a differentially private execution. Despite some unfathomably stunning computational results, there are so far head hindrances not just on what can be practiced with differential security anyway on what can be cultivated with any methodology that guarantees against an all out breakdown in assurance. In every way that really matters all of the figurings discussed in this keep up differential insurance against foes of self-selfassured computational force. Certain counts are computationally genuine, others are gainful. Computational multifaceted nature for the enemy and the figuring are both discussed.

As indicated by the Raghav Bhaskar, Srivatsan Laxman and Adam Smith [4], Private information examination in the setting in which a trusted and reliable guardian, having gotten an enormous informational collection containing private data, discharges to the open a Isanitization" of the informational collection that at the same time secures the protection of the individual givers of information and clients utility to the information examiner. The purification might be as a subjective information structure, joined by a computational strategy for deciding estimated answers to questions on the first informational collection, or it might be an engineered informational collection" comprising of information things drawn from a similar universe as things in the first informational collection; inquiries are completed as though the manufactured informational index were the genuine information. In either case the procedure is non-intelligent; when the cleansing has been discharged the first information and the custodian assume no further job. 
As indicated by the, Cynthia D work and Aaron Roth [5], the issue of security safeguarding information investigation had a long history traversing different controls. As innovation empowers perpetually amazing assortment of these information, the need increments for a hearty, significant, and numerically thorough meaning of protection, together with a computationally high class of calculations that fulfill this definition.

Resulting to convincing and discussing the significance of differential assurance, the pervasiveness of the book is resolved to key methodology for achieving differential security, and utilization of these strategies in innovative blends, using the request release issue as a ceaseless point of reference. A key point is that, by rethinking the computational target, one can consistently obtain much preferable results over would be practiced by methodically superseding every movement of a non-private estimation with a differentially private utilization. Regardless of some unimaginably astonishing computational results, there are so far significant limitations - not just on what can be cultivated with differential security yet on what can be practiced with any system that guarantees against an all out breakdown in insurance.

As per the Trupti Kenekar1, A. R. Dani [6], Visit sets accept an essential activity in various Data Mining tasks that attempt to find captivating models from databases, for instance, association rules, associations, groupings, scenes, classifiers and pack. The ID of sets of things, things, reactions and characteristics, which regularly happen together in the given database, can be seen as a champion among the most essential assignments in Data Mining. The main motivation for looking for progressive sets began from the need to separate implied showcase trade data, that is, to examine customer lead similar to the acquired things. Visit sets of things depict how routinely things are acquired together.

The current framework had issue of tradeoff among utility and security in planning a differentially private FIM calculation. The current framework doesn't manage the high utility value-based thing sets. Existing strategies has enormous time multifaceted nature. Existing framework gives relatively enormous size yield blend. With correspondence, information stockpiling innovation, an immense measure of data is being gathered and put away in the Internet. Information mining, with its guarantee to effectively.

\section{PROBLEM DEFINITION}

Design privately mining high confidence rules, where each transaction contains a set of items, frequent item set mining tries to find that occur more frequently than a given threshold.

\section{OBJECTIVES}

1. A novel technique for differentially private mining of association rules with low and moderate supports.

2. Technique directly samples high confidence rules using the exponential mechanism.

\section{METHODOLOGY}

Followings are some modules here introduced to mining the patterns.

\section{Rule Expansion Optimization}

We present a streamlining that improves the utility of the calculation by creating a larger number of rules than the mentioned $\mathrm{k}$. in particular; the advancement utilizes properties of association rules to induce extra high-certainty rules beginning from the set $\mathrm{Rk}$ of $\mathrm{k}$ rules returned by the calculation.

\section{Enhancements of HCR MINING}

By joining exponential components and repository testing, the HCRMine calculation brings a crucial improvement contrasted with existing private guideline extraction systems. In any case, as the quantity of mentioned rules k expands, the protection spending should be isolated among increasingly exponential instrument summons. Thus, exactness will diminish. We officially break down HCRMine and recognize the reason for accuracy debasement. Next, we present two varieties of HCRMine that address this issue. We propose the HCRBins technique which benefits from the equal creation property of differential security, and performs rule extraction on disjoint segments (or containers) of things.

\section{HCRMINE}

The use of the exponential system requires the calculation of the quality capacities for every up-and-comer rule. Given a lot of $n$ things, the absolute number of decides that can be created is $3 n-2 n+1+1$. Extricating k rules from this set has computational multifaceted nature $\mathrm{O}(\mathrm{k} \times 3 \mathrm{n})$ (the quality capacity must be figured for every competitor in every one of the $\mathrm{k}$ exponential system execution adjusts). This overhead is restrictive in any event, for moderate estimations of $n$. We mean to bring the computational multifaceted nature of private high-certainty rules to handy levels. Besides, so as to utilize the security spending plan sensibly, we have to guarantee that we don't produce a similar guideline on different occasions. To accomplish this, each time we slide the window of qualified things, we generally produce decides that contain the recently included thing. For example, if the arrangement of qualified things changes from $\{\mathrm{i} 1, \mathrm{i} 2, \mathrm{i} 3, \mathrm{i} 4\}$ to $\{\mathrm{i} 2, \mathrm{i} 3, \mathrm{i} 4, \mathrm{i} 5\}$ all the principles that are produced in the new advance must contain thing i5. As a reaction, the multifaceted nature of the standard age is likewise decreased.

\section{Examining HCRMine}

So as to think about two diverse exponential instruments, A1 and $\mathrm{A} 2$, with the equivalent ideal worth frothier individual quality capacity, we just need to break down the capacities $\emptyset \mathrm{A} 1$ and $\varnothing \mathrm{A} 2$. Besides, if the quality capacities are both characterized on subsets of a similar set R0 with the end goal that the two logarithms are around the equivalent.

\section{HCRMINE}

The use of the exponential component requires the calculation of the quality capacities for every up-and-comer rule. Given a lot of $\mathrm{n}$ things, the all out number of decides that can be created is $3 n-2 n+1+1$. Separating $k$ rules from 
this set has computational intricacy $\mathrm{O}(\mathrm{k} \times 3 \mathrm{n})$ (the quality capacity must be registered for every competitor in every one of the $\mathrm{k}$ exponential instrument execution adjusts). This overhead is restrictive in any event, for moderate estimations of $\mathrm{n}$. We mean to bring the computational unpredictability of private high-certainty rules to down to earth levels. Moreover, so as to utilize the security spending plan reasonably, we have to guarantee that we don't create a similar guideline on numerous occasions. To accomplish this, each time we slide the window of qualified things, we generally create decides that contain the recently included thing. For example, if the arrangement of qualified things changes from $\{i 1, i 2, i 3, i 4\}$ to $\{i 2, i 3, i 4, i 5\}$ all the guidelines that are produced in the new advance must contain thing i5. As a symptom, the multifaceted nature of the standard age is additionally decreased.

\section{The HCRBins calculation}

To improve mining precision, we exploit the equal piece property of differential protection. Assume we deteriorate the set I of things into two disjoint subsets, I1 and I2. Moreover, to get $\mathrm{k}$ rules, we remove $\mathrm{k} 1$ rules from the things in $\mathrm{I} 1$ and $\mathrm{k} 2$ rules from the things in $\mathrm{I} 2$.Next, we demonstrate that we don't have to part the security spending plan into two segments, since we have a case of equal arrangement. Without loss of sweeping statement, assume that $t$ is the exchange that is being added or expelled from $\mathrm{D}$ to acquire the neighboring dataset $\mathrm{D}^{\prime}$. We have two potential cases:

- $\mathrm{t} \cap \mathrm{I} 1=\emptyset$. For this situation, rule $\mathrm{r}$ isn't influenced by the evacuation or expansion of $t$ since nothing in $r$ is in $\mathrm{t}$.

Henceforth, $\mathrm{q}(\mathrm{D}, \mathrm{I} 1, \mathrm{r})=\mathrm{q}\left(\mathrm{D}^{\prime}, \mathrm{I} 1, \mathrm{r}\right)$ and the examining likelihood doesn't change.

- $\mathrm{t} \cap \mathrm{I} 16=\varnothing$. The main situation when the estimation of the quality capacity changes is the point at which the standard $r$ is framed uniquely by things in $\mathrm{t} \cap \mathrm{I}$. Since I1 and I 2 are disjoint, the standard won't be considered for testing twice, so the adjustment in quality capacity is limited by its affectability.

\section{CONCLUSION}

Work presents a protection safeguarding approach for noting association rules inquiries to save the two information security and inquiry Confidentiality. The proposed strategy shields induction assaults from information buyers by ensuring that the returned association rules to the information shopper to fulfill. Differential security, to jam the protection of the mined information by confining every datum supplier from learning touchy data about other information suppliers during the mining procedure, next ensures the privacy of the information purchaser's question against the information suppliers to such an extent that the ace excavator can mine the association rules without uncovering the inquiry to the information suppliers.

\section{REFERENCES}

[1] R. Agrawal and R. Srikant. "Privacy preserving data mining", InProceedings of International Conference on Management of Data (ACMSIGMOD), 2000.

[2] R. Bhaskar, S. Laxman, A. Smith, and A. Thakurta "Discoveringfrequent patterns in sensitive data". In Proc. of Intl. Conf. on Knowledge Discovery and Data Mining (KDD), pages 503-512, 2010.

[3] R. Chen, B. C. Fung, B. C. Desai, and N. M. Sossou. "Differentiallyprivate transit data publication: a case study on the Montrealtransportation system”. In Proc. of Intl. Conf. on Knowledge Discoveryand Data Mining (KDD), pages 213-221, 2012.

[4] G. Cormode, C. Procopiuc, E. Shen, D. Srivastava, and T. Yu.'Differentially private spatial decompositions." In ICDE, pages 20 31, 2012.

[5] C. Dwork, F. McSherry, K. Nissim, and A. Smith. "Calibrating noiseto sensitivity in private data analysis". In TCC, pages 265-284, 2006.

[6] C. Dwork, M. Naor, O. Reingold, G. N. Rothblum, and S. Vadhan.”On the complexity of differentially private data release: Efficient algorithms and hardness results". In ACM Symposium on Theory ofComputing, pages 381-390, 2009

[7] C. Dwork and A. Roth. "The algorithmic foundations of differentialprivacy". Foundations and Trends in Theoretical Computer Science,9(34):211-407, 2014.

[8] Friedman and A. Schuster. "Data mining with differentialprivacy". In Proc. of Intl. Conf. on Knowledge Discovery and DataMining (KDD), pages 493-502, 2010.

[9] Ghosh, T. Roughgarden, and M. Sundararajan. "Universally utilitymaximizing privacy mechanisms". In ACM Symposium onTheory of Computing, pages 351-360, 2009

[10] Omar Abdel Wahab, Moulay Omar Hachami etal "DARM: A Privacypreserving Approach for Distributed Association Rules Mining on Horizontally-partitioned Data". Conference Paper · July 2014 DOI: $10.1145 / 2628194.2628206$

[11] Kalas, Mamata \& Unne, Amruta. (2019). High Confidence Association Rule for Product Selling Strategy. International Journal of Computer $\begin{array}{lll}\text { Sciences and } & \text { Engineering. } & \end{array}$ 10.26438/ijcse/v7i6.11841188. 